\title{
Strong Linear Dichroism in Spin-Polarized Photoemission from Spin-Orbit-Coupled Surface States
}

\author{
H. Bentmann, ${ }^{1}$ H. Maaß, ${ }^{1}$ E. E. Krasovskii, ${ }^{2,3,4}$ T. R. F. Peixoto, ${ }^{1}$ \\ C. Seibel, ${ }^{1}$ M. Leandersson, ${ }^{5}$ T. Balasubramanian, ${ }^{5}$ and F. Reinert ${ }^{1}$ \\ ${ }^{I}$ Experimentelle Physik VII and Röntgen Research Center for Complex Materials (RCCM), \\ Universität Würzburg, Am Hubland, D-97074 Würzburg, Germany \\ ${ }^{2}$ Departamento de Física de Materiales, Facultad de Ciencias Quíimicas, \\ Universidad del Pais Vasco/Euskal Herriko Unibertsitatea, \\ Apdo. 1072, San Sebastián/Donostia, 20080 Basque Country, Spain \\ ${ }^{3}$ Donostia International Physics Center (DIPC), Paseo Manuel de Lardizabal 4, \\ San Sebastián/Donostia, 20018 Basque Country, Spain \\ ${ }^{4}$ IKERBASQUE, Basque Foundation for Science, 48013 Bilbao, Spain \\ ${ }^{5}$ MAX IV Laboratory, Lund University, P. O. Box 118, 22100 Lund, Sweden
}

(Dated: May 25, 2022)

\begin{abstract}
A comprehensive understanding of spin-polarized photoemission is crucial for accessing the electronic structure of spin-orbit coupled materials. Yet, the impact of the final state in the photoemission process on the photoelectron spin has been difficult to assess in these systems. We present experiments for the spin-orbit split states in a Bi-Ag surface alloy showing that the alteration of the final state with energy may cause a complete reversal of the photoelectron spin polarization. We explain the effect on the basis of ab initio one-step photoemission theory and describe how it originates from linear dichroism in the angular distribution of photoelectrons. Our analysis shows that the modulated photoelectron spin polarization reflects the intrinsic spin density of the surface state being sampled differently depending on the final state, and it indicates linear dichroism as a natural probe of spin-orbit coupling at surfaces.
\end{abstract}

The creation and manipulation of spin-polarized electronic states in crystalline solids, low-dimensional systems, and heterostructures through strong spin-orbit interaction is a central topic in contemporary condensed matter physics [1-5]. Among the most vivid examples are the surface states of topological insulators in which the coupling of the spin and momentum degrees of freedom gives rise to helical spin textures in momentum space [6]. Moreover, spin-orbit split band structures, in general, attract attention in a broad range of materials, including Weyl semimetals [7], with unconventional spin-polarized states in the bulk and at the surface, stronglycorrelated topological Kondo insulators [8, 9], as well as twodimensional systems, such as metallic oxide interfaces [5] and transition-metal dichalcogenide layers [3]. Thus, given the tremendous interest in spin-orbit coupled materials, it is of critical importance to probe their electronic structure with spin sensitivity and to reliably verify the anticipated spin dependences, see, e.g., Refs. [10-13].

The most versatile tool to spectroscopically address the momentum-dependent spin polarization of electronic band structures in condensed matter physics has been spin- and angle-resolved photoemission spectroscopy (spin-ARPES). In recent years it has been successfully applied to a variety of materials [14-16]. At the same time, the efficiency of stateof-the-art photoelectron spin detectors has been improved tremendously [11, 16, 17], making it now possible to measure the photoelectron spin polarization over wide regions of momentum space with varying energy and polarization of the exciting light. Despite these encouraging developments fundamental issues remain debated, namely to which degree, un- der which conditions, and in which way the measured photoelectron spin polarization actually reflects the intrinsic spin properties of spin-orbit split states [11, 18-26].

Within the one-step theory of photoemission the spindependent photocurrent is determined by the photoemission matrix element which involves the initial state of the transition - the object of interest - and the final state of the outgoing photoelectron. In the vacuum ultraviolet photonenergy regime, commonly used in spin-ARPES experiments, the properties of the final state may quickly vary with the excitation energy and deviate considerably from the naive assumption of a free-electron state [27]. This is well-known to induce pronounced modulations of the spin-integrated photoemission intensity, as observed, e.g., for surface states on metals [28, 29] and on topological insulators [30]. Yet, a comprehensive understanding of how the final state affects the spin-resolved photocurrent of spin-orbit split states remains elusive.

Here, we show in a joint experimental and theoretical study that variation of the final-state wave function with energy can induce a complete reversal of the photoelectron spin. Based on general symmetry considerations and dipole selection rules we relate this surprising reversal in the photoelectron spin polarization to simultaneous modulations in the angle-resolved photoemission intensity. Both effects originate from a strong linear dichroism in the angular distribution of photoelectrons and are confirmed by ab initio one-step photoemission theory. The results are obtained for the surface alloy $\mathrm{BiAg}_{2} / \mathrm{Ag}(111)$, a model system for spin-orbit effects [3134] and spin-dependent photoemission [18, 26, 35-37]. Its 

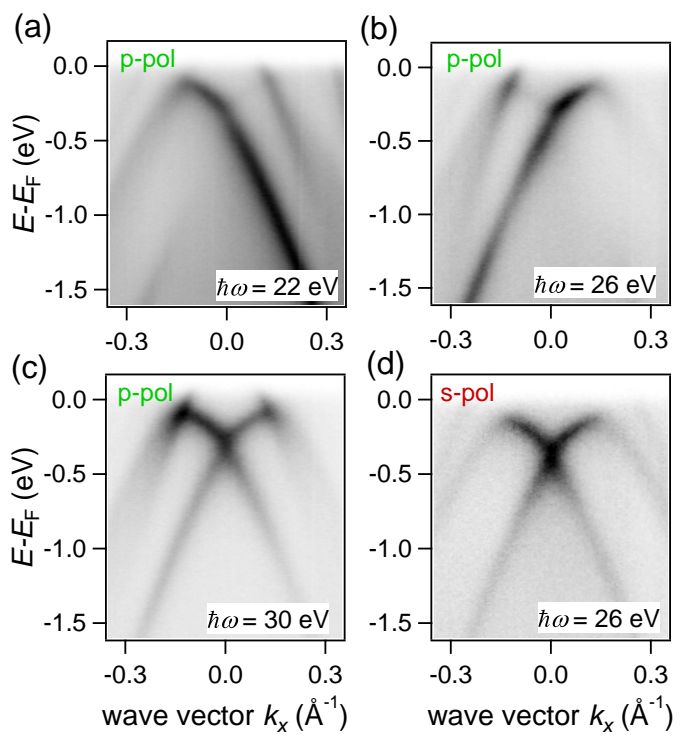

(e)
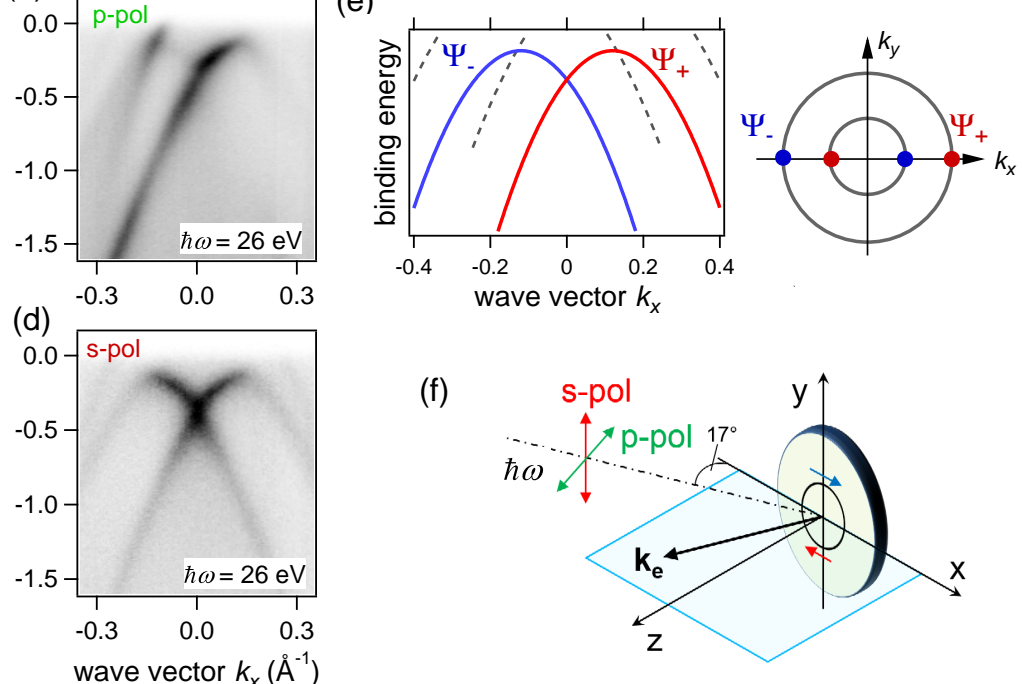

(f)

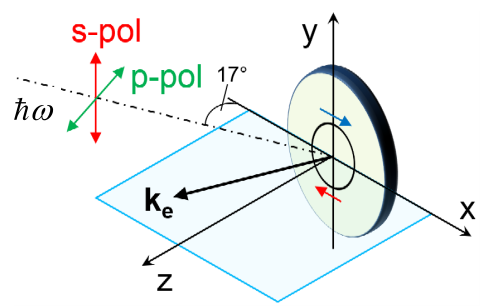

FIG. 1: Angle-resolved photoemission data of the spin-orbit split surface states in $\mathrm{BiAg}_{2} / \mathrm{Ag}(111)$ taken along the $\bar{\Gamma} \bar{M}$ direction $\left(k_{x}\right)$ using $p$-polarized in (a)-(c) and $s$-polarized light in (d). (e) Schematic of the spin-orbit split branches $\Psi_{+}$and $\Psi_{-}$. (f) Sketch of the experimental geometry.

electronic structure features a nearly parabolic, Bi $6 p$-derived band with negative effective mass. Due to large Rashba-type spin-orbit coupling the band is split into two branches $\Psi_{+}$and $\Psi_{-}$with opposite spin polarization [Fig. 1(e)].

The experiments were performed at room-temperature and in ultrahigh vacuum $\left(p<2 \cdot 10^{-10}\right.$ mbar $)$. The surface alloy [31, 35] was grown as described elsewhere [38]. Spin-ARPES experiments were performed at beamline I3 of the Max-lab storage ring (Lund, Sweden). The experimental geometry is depicted in Fig. 1. (c). We used a Scienta R4000 photoelectron analyzer with a Mott-detector operated at $25 \mathrm{kV}$. The energy resolution of the ARPES and spin-ARPES experiments were approximately $15 \mathrm{meV}$ and $50 \mathrm{meV}$, respectively. The angular resolution for spin-ARPES experiments was $3^{\circ}$. The Sherman function of the Th Mott-target was $S_{\text {eff }}=0.17$.

We first consider spin-integrated ARPES data along the $\bar{\Gamma} \bar{M}$ direction $\left(k_{x}\right)$ obtained with $p$-polarized light [Fig. 11(a)-(c)]. The intensities of both branches $\Psi_{+}$and $\Psi_{-}$strongly modulate with photon energy [see also Fig. 2(a) and Fig. S1 in the supplement], in agreement with earlier work [36]. The dependence of the intensity on photon energy differs for $\Psi_{+}$ and $\Psi_{-}$. This leads to pronounced $\pm k_{x}$ asymmetries that can be classified as linear dichroism in the angular distribution of photoelectrons [39]. They are related to the light electric field vector $\mathbf{E}=\left(\mathcal{E}_{x}, 0, \mathcal{E}_{z}\right)$, which breaks the $\pm k_{x}$ mirror symmetry of the experimental setup. By contrast, symmetric intensity distributions are observed when using $s$-polarized light, as seen in Fig. 1 d d) and in Fig. S2 of the supplement.

The ARPES data along $k_{x}$ are largely independent of the azimuthal crystal orientation [Fig. S4 of the supplement]. We may, therefore, assume that the system, besides the mirror symmetry $x \rightarrow-x$, also has the mirror symmetry $y \rightarrow-y$. This is reasonable because the surface states are strongly localized in the alloy layer, which has both mirror reflections. For a non-degenerate state with $\mathbf{k}_{\|}$in the mirror plane $x z$ the wave functions $\Psi_{+}$and $\Psi_{-}$along $k_{x}$, are connected by timereversal symmetry and can be written as $\Psi_{+}=g \chi^{\uparrow}+u \chi^{\downarrow}$ and $\Psi_{-}=u^{*} \chi^{\uparrow}-g^{*} \chi^{\downarrow}$, with $\chi^{\sigma}$ quantized along $y$. The spinor component $g(\mathbf{r})$ is even under the reflection $y \rightarrow-y$, and $u(\mathbf{r})$ is odd [40]. Hence, the net spin density $|g(\mathbf{r})|^{2}-|u(\mathbf{r})|^{2}$ varies with position $\mathbf{r}$ as a consequence of spin-orbit coupling. We then introduce the mirrored functions $\tilde{g}(x, y, z)=$ $g(-x, y, z)$ and $\tilde{u}(x, y, z)=u(-x, y, z)$ and write $\Psi_{-}=$ $\tilde{u} \chi^{\uparrow}+\tilde{g} \chi^{\downarrow}$.

Within the dipole approximation for the photoexcitation operator $\hat{O}$ rigorous parity selection rules hold: because the final state - the time-reversed LEED state $|\Phi\rangle$ - is necessarily even, for $p$-polarized light incident in the emission plane it is $\langle\Phi|\hat{O}| u\rangle=0$, and for $s$-polarized light it is $\langle\Phi|\hat{O}| g\rangle=0$. Hence, for $p$-polarization the photoemission intensities $I_{+}$and $I_{-}$for $\Psi_{+}$and $\Psi_{-}$can be written as

$$
\begin{aligned}
& I_{+}=\left|\left\langle\Phi\left|\mathcal{E}_{z} p_{z}\right| g\right\rangle+\left\langle\Phi\left|\mathcal{E}_{x} p_{x}\right| g\right\rangle\right|^{2} \text { and } \\
& I_{-}=\left|\left\langle\tilde{\Phi}\left|\mathcal{E}_{z} p_{z}\right| \tilde{g}\right\rangle+\left\langle\tilde{\Phi}\left|\mathcal{E}_{x} p_{x}\right| \tilde{g}\right\rangle\right|^{2},
\end{aligned}
$$

where we have neglected the spin-orbit interaction in the final state. Because $T_{z}=\left\langle\Phi\left|\mathcal{E}_{z} p_{z}\right| g\right\rangle=\left\langle\tilde{\Phi}\left|\mathcal{E}_{z} p_{z}\right| \tilde{g}\right\rangle$ and $T_{x}=\left\langle\Phi\left|\mathcal{E}_{x} p_{x}\right| g\right\rangle=-\left\langle\tilde{\Phi}\left|\mathcal{E}_{x} p_{x}\right| \tilde{g}\right\rangle$ we obtain the intensity asymmetry: $I_{+}=\left|T_{z}+T_{x}\right|^{2}$ and $I_{-}=\left|T_{z}-T_{x}\right|^{2}$.

A full suppression of $I_{+}$and $I_{-}$is expected for $I_{z} \approx I_{x}$, with $I_{x, z}=\left|T_{x, z}\right|^{2}$, and for phase differences between $T_{z}$ and $T_{x}$ of $\Delta \phi=\pi$ and $\Delta \phi=0$, respectively. These conditions are met for $\hbar \omega=22 \mathrm{eV}\left(I_{+}=0\right)$ and $\hbar \omega=26 \mathrm{eV}$ 

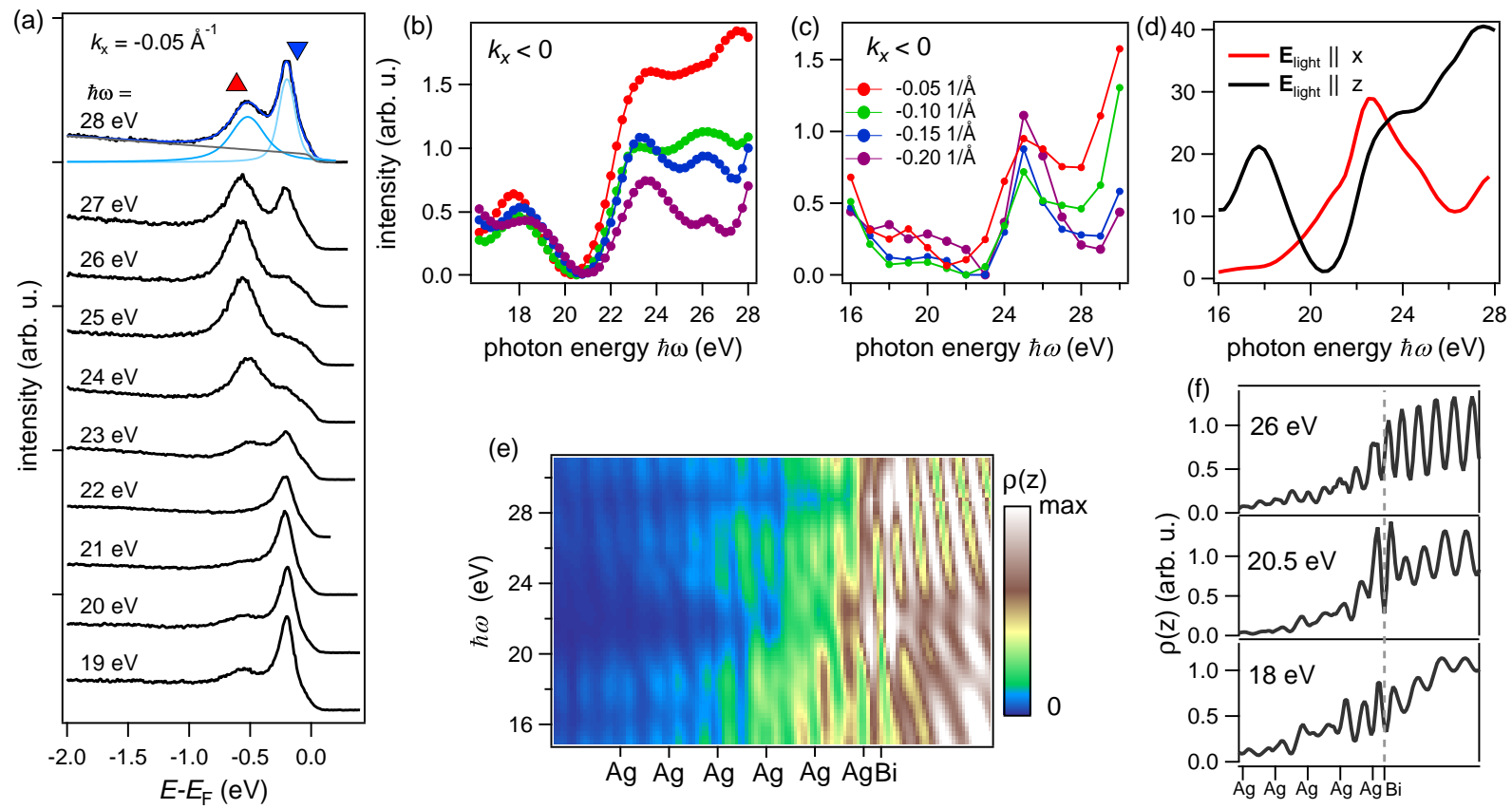

FIG. 2: Modulation of the photoemission intensity of the spin-orbit split branches $\Psi_{+}$and $\Psi_{-}$in $\mathrm{BiAg}_{2} / \mathrm{Ag}(111)$ with photon energy (cf. Fig. 1). Panel (a) shows energy distribution curves (EDC) at negative wave vectors $k_{x}$. The spectra were normalized to the background intensity at binding energies below $1.5 \mathrm{eV}$. The two peaks in each EDC are assigned to the branches $\Psi_{+}$and $\Psi_{-}$. Calculated and measured intensities for $\Psi_{+}$depending on photon energy are shown in (b) and (c). (d) Calculated intensities $I_{z}$ and $I_{x}$ for the light electric field along $z$ and along $x$, respectively, for the branch $\Psi_{+}$at negative $k_{x}$. (e) Probability density $\rho(z)$ of the photoelectron final state $|\Phi\rangle$ as a function of the surface-normal coordinate $z$ and $\hbar \omega$. (f) Line profiles of $\rho(z)$ in (e) at selected photon energies.

( $I_{-}=0$ ) [Fig. [1(a)-(b)]. A simultaneous zero-crossing of $T_{z}$ and $T_{x}$ can be excluded as, in this case, both $I_{+}$and $I_{-}$would be suppressed. Given the grazing angle of light incidence $\left(\mathcal{E}_{z} / \mathcal{E}_{x} \approx 3\right)$ one would naturally assume $I_{z}>I_{x}$, leading to rather symmetric intensity distributions. This is indeed the case for most parts of the studied photon-energy range of $\hbar \omega=8 \ldots 40 \mathrm{eV}$ [Fig. S1 in the supplement]. However, near $\hbar \omega=22 \mathrm{eV}$ and $\hbar \omega=26 \mathrm{eV}$ we find $I_{z} \approx I_{x}$, and in between one may expect $I_{x}>I_{z}$.

In order to go beyond these qualitative considerations we present one-step photoemission calculations of the $\hbar \omega$ dependent intensity. The final state $|\Phi\rangle$ is the time-reversed low energy electron diffraction state [41] calculated for the scattering of electrons on a slab composed of the surface alloy and five layers of $\operatorname{Ag}(111)$ substrate. The potential of the slab was obtained within the local density approximation with the full-potential linear augmented plane wave method (LAPW) [42]. For the initial states the relativistic effects were included within a two-component formalism [43], and the final states were obtained in the scalar relativistic approximation with the inverse LAPW method [44]. A detailed description of the procedure can be found in Ref. [45]. The inelastic scattering of photoelectrons is included by adding a spatially constant imaginary part $V_{\mathrm{i}}=1 \mathrm{eV}$ to the crystal potential.

Figure 2(d) shows the calculated intensities $I_{z}$ and $I_{x}$ of the branch $\Psi_{+}$for light polarized linearly along $z$ and along $x$, respectively. We find markedly different $\hbar \omega$ dependences for $I_{z}$ and $I_{x}$ and, in particular, a pronounced minimum of $I_{z}$ near $\hbar \omega=21 \mathrm{eV}$ as well as a maximum of $I_{x}$ near $\hbar \omega=23 \mathrm{eV}$. As expected from the experimental data, we find a range of photon energies where $I_{x}>I_{z}$.

In Fig. 2.b)-(c) we compare the calculated and measured photoemission intensities of the branch $\Psi_{+}$. The strong intensity variation observed at these energies is remarkably well reproduced by the $a b$ initio theory. It is related to a Bragg gap at approximately $22 \mathrm{eV}$ in the unoccupied band structure of the $\operatorname{Ag}(111)$ substrate, which was first observed in Ref. [46] and analyzed from the band structure point of view in Ref. [44]. Because the initial states are strongly localized in the surface layer, the strong decay of the final state due to the gap in itself can hardly affect the intensity. Nevertheless, at energies of the gap the propagation of the outgoing photoelectron proceeds differently, which is illustrated by the energy-depth distribution of the probability density $\rho(z)$ in the final state $|\Phi\rangle$ in Fig. 2(e). One can see a scattering resonance near $22 \mathrm{eV}$ that is also visible in the line profile at $20.5 \mathrm{eV}$ in Fig. 2ff). The rapid modification of the final state in approaching the resonance gives rise to the observed intensity modulations. Notice, in particular, the nearly symmetric behavior of $\rho(z)$ around the $\mathrm{Bi}$ atom at around $20.5 \mathrm{eV}$ where the intensity $I_{z}$ drops to zero. 

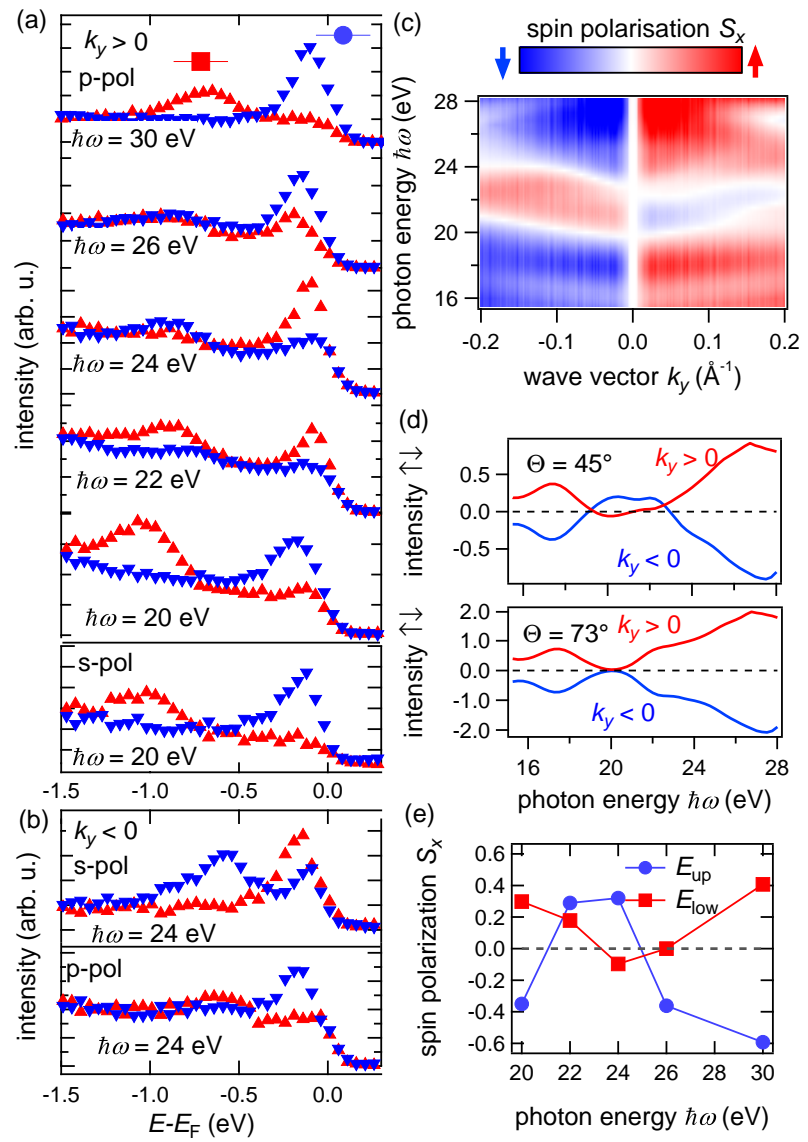

FIG. 3: Modulation of the photoelectron spin polarization in $\mathrm{BiAg}_{2} / \mathrm{Ag}(111)$ with excitation energy $\hbar \omega$. (a)-(b) Spin-resolved EDC at positive (a) and negative (b) wave vectors along $k_{y}$ [cf. Fig. 1.e)-(f)]. The spin-quantization axis of the measurement is aligned with the $x$-axis. (c) One-step photoemission calculation of the photoelectron spin polarization for the branch at lower energy along $k_{y}$ assuming $p$-polarized light and an angle of incidence $\theta=45^{\circ}$. (d) Calculated net-spin photocurrents of the branch at lower energy at $k_{y}= \pm 0.1 \AA^{-1}$ for $\theta=45^{\circ}$ and $\theta=73^{\circ}$ (e) Measured photoelectron spin polarization for the spin-orbit split branches depending on $\hbar \omega$, as extracted from the data in (a).

We will now show how the variations of $T_{z}$ and $T_{x}$ affect the photoelectron spin polarization. Along the $k_{y}$ direction the wave function $\Psi_{+}$[Fig. 1] reads $\Psi_{+}=g \chi^{\uparrow}+u \chi^{\downarrow}$, with $g(\mathbf{r})$ and $u(\mathbf{r})$ being even and odd upon reflection $x \rightarrow-x$, respectively, and $\chi^{\sigma}$ quantized along $x$. In this geometry, the intensity $I_{z}$ corresponds to spin- $\uparrow$ and the intensity $I_{x}$ to spin$\downarrow$ photoelectrons, because $\mathcal{E}_{z}$ couples to $g(\mathbf{r})$ and $\mathcal{E}_{x}$ to $u(\mathbf{r})$. Hence, we expect the photoelectron spin polarization $P_{x}$ to have opposite sign for $I_{z}>I_{x}$ and $I_{z}<I_{x}$.

This is confirmed by the spin-resolved data in Fig. 3 (3). The measured polarization flips sign twice as a function of $\hbar \omega$ [Fig. 3. (e)], confirming that $I_{z}-I_{x}$ changes sign as expected from the spin-integrated data. Additional insight is gained from data acquired with $s$-polarized light in Fig. 3 (a)-(b). Along $k_{y}, s$-polarized light only couples to the even part $g(\mathbf{r})$ of $\Psi_{+}$, as does the $z$-component of $p$-polarized light. Thus, if $I_{z}-I_{x}>0$ the sign of the photoelectron spin should coincide for $s$ - and for $p$-polarized light, whereas opposite signs are expected if $I_{z}-I_{x}<0$. We find the former at $\hbar \omega=20 \mathrm{eV}$ and the latter at $\hbar \omega=24 \mathrm{eV}$ [Fig. 3 (3)-(b)], which implies $I_{x}>I_{z}$ between approximately $\hbar \omega=22 \mathrm{eV}$ and $26 \mathrm{eV}$.

Our one-step photoemission calculations of the photoelectron spin polarization in Figure 3 (c) confirm the sign change. We find that the spin polarization depends on the angle of light incidence $\theta$, because the relative magnitudes of $\mathcal{E}_{x}$ and $\mathcal{E}_{z}$ vary with $\theta$ [Fig. 3 d)]. The spin polarization changes sign for $\theta=45^{\circ}$ but for $\theta=73^{\circ}$ it merely dips to 0 . In the experiment a sign change is observed at $\theta=73^{\circ}$. This discrepancy likely arises from uncertainties concerning the actual exciting field at the surface: the dielectric response is rather large at around $\hbar \omega=21 \mathrm{eV}$, so that the electric field at the surface may differ both from the field in the vacuum and from the field in the depth of the crystal [47]. However, in the studied $\hbar \omega$-range, the real part of the dielectric function $\epsilon_{1}$ of $\mathrm{Ag}$ is positive and shows a smooth behavior [48], so that we expect no qualitative influence of the dielectric response on the observed photon-energy dependence.

In conclusion, we demonstrated that the photoelectron spin polarization of a spin-orbit-split surface state can strongly modulate and fully reverse upon only small changes in the excitation energy $(\delta \omega / \omega<10 \%)$. Such a strong effect of the variation of the final state with energy will be of importance for spin-ARPES experiments on various spin-orbit coupled materials, such as topological insulators, Weyl semimetals, and Rashba systems. In contrast to previous photoemission studies, which focused either on the spin polarization or on the intensity (e.g., circular dichroism), here the relation between the two observables, originating from the dipole selection rules, unambiguously points to the origin of the effect. Our findings, thereby, challenge the claim of a negligible role of the dipole operator stated in a previous work on the same material [37].

Remarkably, our theory reproduces the nontrivial photonenergy-dependence of both the intensity and the spin polarization of the photocurrent. It also explains their origin as due to modulations of the final-state wave function at a Bragg gap in the unoccupied band structure. Details of the final state wave function are seen to have a major effect on the spin photocurrent even when spin-dependent scattering of the outgoing photoelectron does not lead to a spin polarization, that is when spin-orbit coupling in the final state is neglected. The latter effect - spin rotation in the course of propagation [49] - is known, both experimentally and theoretically, to be appreciable at kinetic energies of a few $\mathrm{eV}$, but at small angles it rapidly vanishes at higher energies [50]. By contrast, the modulated photoelectron spin polarization observed in this work reflects the intrinsic spin properties of the probed state, namely its spatially varying spin density, which is sampled differently depending on the shape of the final-state wave function. This is in contrast to the seemingly similar photon- 
energy-dependence of the circular dichroism in related systems [30].

It is common belief that dichroic photoemission is an indispensable source of information about the spinor wave function. The vast majority of previous works considered circular dichroism, and a variety of interpretations have been put forward that related it to the spin [51] or to a local orbital angular momentum [52] in the initial state or to a final-state effect [30]. No consensus appears to be reached as to how the circular dichroism reflects the properties of the relativistic wave function. By contrast, the linear dichroism we report has a clear interpretation and originates from the symmetry properties of the spin-orbit coupling in the initial state in combination with the asymmetry of the experimental geometry. Hence, our study reinforces linear dichroism -as opposed to circular dichroism- as a natural and efficient probe of spinorbit coupling in the band structures of solids and surfaces [39, 53].

\section{ACKNOWLEDGMENTS}

We gratefully acknowledge collaboration with Peter Krüger, and thank Christian Ast, Simon Moser, and Hugo Dil for helpful discussions. This work was supported by the DFG through SFB1170 'Tocotronics' (Project A01) and by the Spanish Ministry of Economy and Competitiveness MINECO (Project No. FIS2016-76617-P).

[1] M. König, S. Wiedmann, C. Brüne, A. Roth, H. Buhmann, L. W. Molenkamp, X. Qi, and S. Zhang, Science 318, 766 (2007).

[2] M. Z. Hasan and C. L. Kane, Rev. Mod. Phys. 82, 3045 (2010).

[3] X. Xu, W. Y. Di Xiao, and T. F. Heinz, Nat. Phys. 10, 343 (2014).

[4] A. Manchon, H. C. Koo, J. Nitta, S. M. Frolov, and R. A. Duine, Nat. Mater. 14, 871 (2015).

[5] E. Lesne, Y. Fu, S. Oyarzun, J. C. Rojas-Sanchez, D. C. Vaz, H. Naganuma, G. Sicoli, J.-P. Attane, M. Jamet, E. Jacquet, et al., Nat. Mater. 15, 12611266 (2016).

[6] D. Hsieh, Y. Xia, L. Wray, D. Qian, A. Pal, J. H. Dil, J. Osterwalder, F. Meier, G. Bihlmayer, C. L. Kane, et al., Science 323, 919 (2009).

[7] S.-Y. Xu, I. Belopolski, N. Alidoust, M. Neupane, G. Bian, C. Zhang, R. Sankar, G. Chang, Z. Yuan, C.-C. Lee, et al., Science 349, 613 (2015).

[8] M. Dzero, K. Sun, V. Galitski, and P. Coleman, Phys. Rev. Lett. 104, 106408 (2010).

[9] C.-H. Min, P. Lutz, S. Fiedler, B. Y. Kang, B. K. Cho, H.D. Kim, H. Bentmann, and F. Reinert, Phys. Rev. Lett. 112, 226402 (2014).

[10] S.-Y. Xu, Y. Xia, L. A. Wray, S. Jia, F. Meier, J. H. Dil, J. Osterwalder, B. Slomski, A. Bansil, H. Lin, et al., Science 332, 560 (2011).

[11] C. Jozwiak, C.-H. Park, K. Gotlieb, C. Hwang, D.-H. Lee, S. G.
Louie, J. D. Denlinger, C. R. Rotundu, R. J. Birgeneau, Z. Hussain, et al., Nat. Phys. 9, 293 (2013).

[12] S. D. Stolwijk, A. B. Schmidt, M. Donath, K. Sakamoto, and P. Krüger, Phys. Rev. Lett. 111, 176402 (2013).

[13] J. M. Riley, F. Mazzola, M. Dendzik, M. Michiardi, T. Takayama, L. Bawden, C. Granerod, M. Leandersson, T. Balasubramanian, M. Hoesch, et al., Nature Phys. 10, 835 (2014).

[14] J. H. Dil, Journal of Physics: Condensed Matter 21, 403001 (2009).

[15] U. Heinzmann and J. H. Dil, Journal of Physics: Condensed Matter 24, 173001 (2012).

[16] T. Okuda and A. Kimura, J. Phys. Soc. Jpn. 82, 021002 (2013).

[17] C. Tusche, A. Krasyuk, and J. Kirschner, Ultramicroscopy 159, 520 (2015).

[18] S. N. P. Wissing, A. B. Schmidt, H. Mirhosseini, J. Henk, C. R. Ast, and M. Donath, Phys. Rev. Lett. 113, 116402 (2014).

[19] Z.-H. Zhu, C. Veenstra, S. Zhdanovich, M. Schneider, T. Okuda, K. Miyamoto, S.-Y. Zhu, H. Namatame, M. Taniguchi, M. Haverkort, et al., Physical Review Letters 112, 076802 (2014).

[20] J. Sanchez-Barriga, A. Varykhalov, J. Braun, S.-Y. Xu, N. Alidoust, O. Kornilov, J. Minár, K. Hummer, G. Springholz, G. Bauer, et al., Physical Review X 4, 011046 (2014).

[21] H. Wortelen, H. Mirhosseini, K. Miyamoto, A. B. Schmidt, J. Henk, and M. Donath, Phys. Rev. B 91, 115420 (2015).

[22] E. E. Krasovskii, Journal of Physics: Condensed Matter 27, 493001 (2015).

[23] C. Seibel, J. Braun, H. Maaß, H. Bentmann, J. Minár, T. V. Kuznetsova, K. A. Kokh, O. E. Tereshchenko, T. Okuda, H. Ebert, et al., Phys. Rev. B 93, 245150 (2016).

[24] K. Miyamoto, H. Wortelen, H. Mirhosseini, T. Okuda, A. Kimura, H. Iwasawa, K. Shimada, J. Henk, and M. Donath, Phys. Rev. B 93, 161403 (2016).

[25] H. Maaß, H. Bentmann, C. Seibel, C. Tusche, S. V. Eremeev, T. R. F. Peixoto, O. E. Tereshchenko, K. A. Kokh, E. V. Chulkov, J. Kirschner, et al., Nature Communications 7, 11621 (2016).

[26] R. Noguchi, K. Kuroda, K. Yaji, K. Kobayashi, M. Sakano, A. Harasawa, T. Kondo, F. Komori, and S. Shin, Phys. Rev. B 95, 041111 (2017).

[27] E. E. Krasovskii, V. N. Strocov, N. Barrett, H. Berger, W. Schattke, and R. Claessen, Phys. Rev. B 75, 045432 (2007).

[28] M. Mulazzi, G. Rossi, J. Braun, J. Minár, H. Ebert, G. Panaccione, I. Vobornik, and J. Fujii, Phys. Rev. B 79, 165421 (2009).

[29] J. Lobo-Checa, J. E. Ortega, A. Mascaraque, E. G. Michel, and E. E. Krasovskii, Phys. Rev. B 84, 245419 (2011).

[30] M. R. Scholz, J. Sánchez-Barriga, J. Braun, D. Marchenko, A. Varykhalov, M. Lindroos, Y. J. Wang, H. Lin, A. Bansil, J. Minár, et al., Phys. Rev. Lett. 110, 216801 (2013).

[31] C. R. Ast, J. Henk, A. Ernst, L. Moreschini, M. Falub, D. Pacilé, P. Bruno, K. Kern, and M. Grioni, Phys. Rev. Lett. 98, 186807 (2007).

[32] H. Bentmann, S. Abdelouahed, M. Mulazzi, J. Henk, and F. Reinert, Phys. Rev. Lett. 108, 196801 (2012).

[33] L. El-Kareh, P. Sessi, T. Bathon, and M. Bode, Phys. Rev. Lett. 110, 176803 (2013).

[34] S. Schirone, E. E. Krasovskii, G. Bihlmayer, R. Piquerel, P. Gambardella, and A. Mugarza, Phys. Rev. Lett. 114, 166801 (2015).

[35] F. Meier, H. Dil, J. Lobo-Checa, L. Patthey, and J. Osterwalder, Phys. Rev. B 77, 165431 (2008).

[36] F. Meier, J. H. Dil, and J. Osterwalder, New Journal of Physics 11, 125008 (2009).

[37] G. Bian, L. Zhang, Y. Liu, T. Miller, and T.-C. Chiang, Phys. 
Rev. Lett. 108, 186403 (2012).

[38] L. Moreschini, A. Bendounan, H. Bentmann, M. Assig, K. Kern, F. Reinert, J. Henk, C. R. Ast, and M. Grioni, Phys. Rev. B 80, 035438 (2009).

[39] J. Henk, M. Hoesch, J. Osterwalder, A. Ernst, and P. Bruno, Journal of Physics: Condensed Matter 16, 7581 (2004).

[40] J. Henk, A. Ernst, and P. Bruno, Phys. Rev. B 68, 165416 (2003).

[41] I. Adawi, Phys. Rev. 134, A788 (1964).

[42] E. E. Krasovskii, F. Starrost, and W. Schattke, Phys. Rev. B 59, 10504 (1999).

[43] D. D. Koelling and B. N. Harmon, J. Phys. C 10, 3107 (1977).

[44] E. E. Krasovskii and W. Schattke, Phys. Rev. B 59, R15609 (1999).

[45] P. Borghetti, J. Lobo-Checa, E. Goiri, A. Mugarza, F. Schiller, J. E. Ortega, and E. E. Krasovskii, J. Phys.: Condens. Matter 24, 395006 (2012).

[46] R. C. Jaklevic and L. C. Davis, Phys. Rev. B 26, 5391 (1982).
[47] E. E. Krasovskii, V. M. Silkin, V. U. Nazarov, P. M. Echenique, and E. V. Chulkov, Phys. Rev. B 82, 125102 (2010).

[48] H.J. Hagemann, W. Gudat, C. Kunz, J. of the Opt. Soc. of America 65, 742 (1975).

[49] H. P. Oepen, K. Hünlich, and J. Kirschner, Phys. Rev. Lett. 56, 496 (1986).

[50] S. N. Samarin, J. F. Williams, A. D. Sergeant, O. M. Artamonov, H. Gollisch, and R. Feder, Phys. Rev. B 76, 125402 (2007).

[51] Y. H. Wang, D. Hsieh, D. Pilon, L. Fu, D. R. Gardner, Y. S. Lee, and N. Gedik, Phys. Rev. Lett. 107, 207602 (2011).

[52] S. R. Park, J. Han, C. Kim, Y. Y. Koh, C. Kim, H. Lee, H. J. Choi, J. H. Han, K. D. Lee, N. J. Hur, et al., Phys. Rev. Lett. 108, 046805 (2012).

[53] W. Kuch and C. M. Schneider, Reports on Progress in Physics 64, 147 (2001). 